\title{
Exploring the effects of omitted variable bias in physics education research
}

\author{
Cole Walsh $\odot,{ }^{1, *}$ Martin M. Stein $\odot,{ }^{1}$ Ryan Tapping $\odot,{ }^{1}$ Emily M. Smith $\odot,{ }^{1,2}$ and N. G. Holmes $\odot^{1}$ \\ ${ }^{1}$ Laboratory of Atomic and Solid State Physics, Cornell University, Ithaca, New York 14853, USA \\ ${ }^{2}$ Department of Physics, Colorado School of Mines, Golden, Colorado 80401, USA
}

(Received 15 February 2020; revised 18 November 2020; accepted 19 February 2021; published 25 March 2021)

\begin{abstract}
Omitted variable bias occurs in most statistical models. Whenever a confounding variable that is correlated with both dependent and independent variables is omitted from a statistical model, estimated effects of included variables are likely to be biased due to omitted variables. This issue is particularly problematic in physics education research where many research studies are quasiexperimental or observational in nature due to ethical and logistical limitations. In this paper, we illustrate the mechanisms behind omitted variable bias in explanatory modeling using authentic data and analytical solutions. We demonstrate that omitting confounding variables that are strongly correlated with included variables and have large effects on the dependent variable can significantly bias estimated effects for included variables. We also find that controlling for variables that are uncorrelated with other variables or have no effect on the dependent variable does not appreciably bias estimated effects and may or may not affect the precision of those estimates. These results suggest that removing from explanatory models variables that are not "statistically significant" can have unintended consequences on model and variable interpretations. Our results underscore the importance of carefully considering why or why not to include a variable in a model, informed by both data and theory.
\end{abstract}

DOI: 10.1103/PhysRevPhysEducRes.17.010119

\section{INTRODUCTION}

Quantitative research in physics education often involves comparing outcome measures across other variables including modes of instruction and student characteristics. A foundational principle of science is that to test the effect of a single variable, we need to hold all other variables constant. In physics education research (PER), or human subjects research in general, controlling all other variables is, for all intents and purposes, impossible. While large sample sizes and random assignment are generally assumed to balance out the variability between conditions, failing to account for differences between conditions can lead to an effect called omitted variable bias [1]. In this paper, we describe omitted variable bias in the context of PER and use student performance data on an attitudinal survey to demonstrate how omitted variable bias manifests. We conclude with recommendations for statistically controlling for variables when experimental control is not feasible.

In PER, like many science and social science fields, statistical methods are used primarily for testing causal hypotheses [2] such as, "how effective is a given pedagogy

*jw295@cornell.edu

Published by the American Physical Society under the terms of the Creative Commons Attribution 4.0 International license. Further distribution of this work must maintain attribution to the author(s) and the published article's title, journal citation, and DOI. in developing student content knowledge?" Shmueli define this type of modeling, using statistical models to test causal explanations, as explanatory modeling [2]. This type of modeling differs from predictive modeling, where a statistical model is used "for the purpose of predicting new or future observations" [2]. We refer the reader to Ref. [2] for a comprehensive review of these two modeling approaches, including study design, variable selection, model selection, and model reporting. Here we focus exclusively on the role of omitted variable bias in explanatory modeling.

Randomized controlled trials (RCTs) are ideal for explanatory modeling, approaching (though not quite achieving) the fundamental control-of-variables experiment we learn about in school. In PER, RCTs are often impractical or unethical to conduct. Many studies (including many of our own) instead use quasiexperimental approaches, where subjects are exposed nonrandomly to an intervention (e.g., mode of instruction).

Consider a study to answer our hypothetical research question above. We may implement a new pedagogy in one semester and compare performance to historical data. Alternatively, we may use observational approaches, where no comparison is made at all. We may implement a new pedagogy in one semester and evaluate student performance on its own or evaluate survey data to understand students' experiences with the new pedagogy. Both of these types of studies can be used to make causal claims, but require stronger assumptions about how data were 
generated, namely that something called ignorability is satisfied [1].

Ignorability is an assumption that subjects are randomly distributed across the variable of interest [1,3]. Ignorability is generally satisfied in RCTs but not in quasiexperimental or observational investigations. Studies do not need to satisfy ignorability to make causal claims. They should, however, satisfy conditional ignorability, where subjects are randomly distributed across the variable of interest conditioned on other observed variables $[1,3,4]$. We can think of conditioning on other variables, practically, as binning our data according to those variables.

Consider a highly simplistic example using our quasiexperimental scenario from above. Suppose there were more physics majors in the semester that the new pedagogy was implemented than in prior semesters. Students, then, were not randomly distributed across modes of instruction. If we binned students according to major and could conclude that within the physics majors group and the nonphysics majors group students were randomly distributed across modes of instruction, then we would satisfy conditional ignorability. In PER these ignorability assumptions are difficult or impossible to check; we cannot observe or even identify all the possible variables about our students. Violation of the conditional ignorability assumption results in omitted variable bias, where the effects of missing variables are attributed to observed variables [1]. We discuss in this paper the specific situations that create omitted variable bias and how this bias can affect results and interpretations in PER.

Previous work in PER examined the specific case of the violation of ignorability for missing data where data that are missing completely at random or missing at random satisfy the strong and conditional ignorability assumptions, respectively [5-7]. Other work investigated omitted variable bias due to confounding variables - variables omitted from analysis that the variable of interest is conditioned on-in specific situations. Several studies, for example, found that students' scientific reasoning ability [8-12], their prior preparation [13-15], their self-efficacy [14], their major [16], or biases inherent in the assessment instruments [13] are confounding variables in analyses of students' performance on standardized assessments. In each of these cases, performance differences between conditions or subgroups of students previously observed were instead at least partially explained by considering an additional, previously omitted variable. We note, however, that in many cases performance differences across demographic variables persist [13,14,16-23].

Many studies do not account for omitted variable bias (arguably, we can never fully account for it) and, as will be demonstrated below, conclusions that attribute effects to a particular variable may be misleading. We use an example from our own work to elaborate. In one study [25], we used random assignment to evaluate the effect of a hands-on, computer simulation, or virtual reality (VR) simulation activity on student learning about moon phases. We found that male students learned better from the VR condition than from the other two conditions and scored higher than the female participants, as measured by a content assessment given before and after the activity. From that result, we may have come to any number of conclusions about biological or sociological differences in men's and women's abilities to learn from VR. Upon closer investigation, however, we identified an important omitted variable: video game experience. Nearly all the male participants in the VR condition had significant video game experience, while nearly all the female participants in the VR condition indicated having very little to no video game experience. This difference was not as stark in the other conditions. Running the analysis with video game experience in lieu of gender showed that video game experience produced the same outcomes as gender in the VR condition. Thus, video game experience was an omitted variable that strongly correlated with the variable of interest, gender, and was not evenly distributed among the conditions.

In this paper, we use real PER data and simple analytic models to demonstrate, quantitatively, what omitted variable bias is, how omitted variables cause bias, and what we can do to mitigate this bias. Throughout, we consider the following hypothetical research question:

What is the causal effect of a students' intended major on their beliefs about experimental physics?

\section{A. Methodological considerations}

We introduce omitted variable bias into our models anytime we specify a model that is not the "true" model. We intentionally use the term "true" in quotations throughout this paper in a hypothetical sense as in practice, we rarely, if ever, know the "true" model, and the actual modeling process involves choosing a set of relevant variables from a much larger set of potentially relevant variables [26]. In explanatory modeling, variables are typically chosen based on the variables' role in the theoretical causal model [2]. The models presented in this paper were built without regard for causal structure and we eschewed careful variable selection, as suggested in Ref. [2], to avoid the estimated effects from our models being interpreted as meaningful. We instead built models based on hypothetical causal models that are not based in any theory or prior work, which allowed us to demonstrate the effect of omitted variable bias. Though the effects estimated from our models are not meaningful, the methodological implications of our results are.

We use linear regression and hypothetical linear models in both Secs. II and III because regression models are the most common form of explanatory modeling seen in the literature [2]. To address our hypothetical research question, we construct models that treat students' attitudes and 
beliefs about experimental physics following instruction (i.e., post-test score) as the dependent variable. Alternative research questions and models, such as using raw gain as the dependent variable for evaluating questions about equity of learning [24], would have been equally valid for illustrating the effects discussed in this paper. We refer the reader to Ref. [27] for a larger discussion about the choice of analysis method for particular research questions. Any type of analysis is prone to omitted variable bias, however, and the important idea is that ignorability is an assumption of the experimental design, not any particular analysis tool.

We make a number of other methodological choices when working with real data in Sec. III that should not be carried forward in authentic studies. Namely, we keep only complete data for analysis despite calls for better handling of missing data in PER [5]. We also do not use hierarchical models to fit our dataset even though this class of models makes more sense theoretically for our dataset with students nested within classes [2,28]. We acknowledge these methodological flaws here to reiterate the intentions of this study. We were not actually interested in answering our hypothetical research question. We were only interested in demonstrating the effect of omitted variable bias. For this reason, our results should not be included in any future meta-analyses.

\section{CONDITIONS FOR OMITTED VARIABLE BIAS}

The magnitude and direction of omitted variable bias depends on the correlation between omitted variables and the included variables, and the "true" effect of omitted variables on the dependent variable. A variable included in a model will be biased by an omitted variable if the following two conditions are met [26]:

(1) the correlation between the omitted variable and the included variable is nonzero, and

(2) the "true" effect of the omitted variable on the dependent variable is nonzero.

For our hypothetical research question proposed in Sec. I, suppose that a simple "true" model exists that relates a student's beliefs about experimental physics following instruction to three variables: the student's beliefs about experimental physics prior to instruction, the student's intended major, and some other variable, $X$ :

post $=\beta_{0}+\beta_{1} \times$ major $+\beta_{2} \times$ pre $+\beta_{3} \times X+\epsilon\left(0, \sigma^{2}\right)$.

In this model, $\beta_{1}, \beta_{2}$, and $\beta_{3}$ are the "true" effects of major, students' prior beliefs about experimental physics, and $X$, respectively, on students' beliefs about experimental physics following instruction. $X$ could be students' self-efficacy, motivation, or something else entirely. All other hypothetical variables not included in Eq. (1) have no effect on students' beliefs about experimental physics by construction.

We evaluate the effect of omitted variable bias by omitting variable $X$ from our analysis and fitting an underspecified model:

$$
\text { post }=\hat{\beta}_{0}+\hat{\beta}_{1} \times \text { major }+\hat{\beta}_{2} \times \text { pre }+\epsilon^{*} \text {, }
$$

where $\epsilon^{*}=\epsilon\left(0, \sigma^{2}\right)+\beta_{3} \times X$ (again, by construction). The expectation value of $\hat{\beta}_{1}$ when fitting this model using ordinary least squares is [26]

$$
\mathbb{E}\left[\hat{\beta}_{1}\right]=\beta_{1}+\beta_{3}\left(\frac{r_{13}-r_{12} r_{23}}{1-r_{12}^{2}} \sqrt{\frac{V_{X}}{V_{\text {major }}}}\right),
$$

where $r_{12}, r_{13}$, and $r_{23}$ are the Pearson correlation coefficients between major and pre, major and $X$, and pre and $X$, respectively, and $V_{\text {major }}$ and $V_{X}$ are the variances of the variables major and $X$. Equation (3) applies only to the case we have here where the "true" model contains three variables, and the underspecified model omits one variable. Variations of Eq. (3) can be derived for any combination of "true" and underspecified models and will generally depend on the "true" effect sizes, correlations between all variables (both included and omitted), and the spread of the data.

Without loss of generality, we assume $V_{X}=V_{\text {major }}$ and rewrite Eq. (3) in terms of the expected bias on $\beta_{1}$ :

$$
\mathbb{E}\left[\hat{\beta}_{1}\right]-\beta_{1}=\beta_{3}\left(\frac{r_{13}-r_{12} r_{23}}{1-r_{12}^{2}}\right) .
$$

The left-hand-side of Eq. (4) (the expected bias) is the difference between the expected estimated regression coefficient for major $\left(\hat{\beta}_{1}\right)$ and the "true" effect of major $\left(\beta_{1}\right)$. A positive value for the expected bias would indicate an overestimation of the effect of major, while a negative value would indicate an underestimation. Note that we did not explicitly examine the bias on $\beta_{2}$ in this analysis, focusing instead on the bias on a single variable. An analogous expression for the expected bias on $\beta_{2}$ can be obtained by swapping the subscripts in Eq. (4) (i.e., $1 \rightarrow 2$ and $2 \rightarrow 1$ ), assuming again that the variances are equal for the two variables.

We illustrate in Fig. 1 the expected bias on $\beta_{1}$ as a function of the "true" effect size of $X$. We use four different plausible combinations of correlation coefficients to show the various levels of omitted variable bias:

(1) pre, major, and $X$ are all weakly correlated $\left(r_{12}=\right.$ $r_{13}=r_{23}=0.2$ )

(2) pre, major, and $X$ are all moderately correlated $\left(r_{12}=r_{13}=r_{23}=0.4\right)$, 
$-r_{12}=r_{13}=r_{23}=0.2-r_{12}=r_{13}=r_{23}=0.4$

$-r_{12}=r_{23}, r_{13}=-0.2-r_{12}=r_{23}=0.2 r_{13}=0.6$

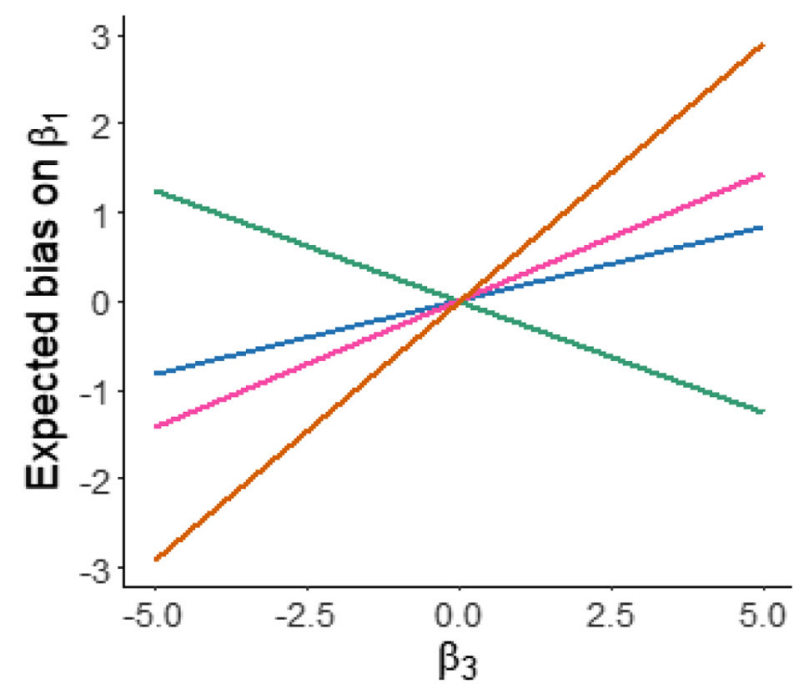

FIG. 1. Expected bias on $\beta_{1}$ (i.e., $\mathbb{E}\left[\hat{\beta_{1}}\right]-\beta_{1}$ ) as a function of $\beta_{3}$ for four different combinations of correlation coefficients, $r_{12}$, $r_{13}$, and $r_{23}$.

(3) pre is weakly correlated with major and $X$, but major and $X$ are weakly anticorrelated $\left(r_{12}=r_{23}=0.2\right.$, $\left.r_{13}=-0.2\right)$,

(4) pre is weakly correlated with major and $X$, but major and $X$ are strongly correlated $\left(r_{12}=r_{23}=0.2\right.$, $\left.r_{13}=0.6\right)$.

Equation (4) indicates that the expected bias is linear in the "true" effect size, $\beta_{3}$ of the omitted variable, $X$. For each of the lines in Fig. 1, the stronger the effect of the omitted variable on the outcome variable (larger $\beta_{3}$ ), the larger the magnitude of the bias. Because the expected bias is linear in $\beta_{3}$, the bias is zero whenever $\beta_{3}$ is zero (i.e., the omitted variable does not affect the outcome variable).

Equation (4) also depends on the correlations between each of the variables. When $X$ is uncorrelated with the other variables in the model (i.e., $r_{13}=r_{23}=0$ ), there is no bias on either $\beta_{1}$ or $\beta_{2}$, reiterating our conditions from above that omitting a variable will only bias coefficient estimates if that variable is correlated with other variables in the presented model. When $\beta_{3}$ is nonzero and $X$ is correlated with at least one of the remaining variables, the magnitude of the expected bias generally increases with the magnitude of the correlations between variables. This bias is shared between the remaining variables based on how strongly the variables are correlated with the omitted variable. Variables that are more strongly correlated with an omitted variable are typically biased to a greater extent, though this is not always the case. The expected bias on $\beta_{1}$ in our scenario, for example, is zero when $r_{13}=r_{12} r_{23}$. The signs of the correlations are important for dictating the direction of the bias, as shown in Fig. 1 for the line where major and $X$ are anticorrelated.
In this analysis, we have examined the absolute bias on $\beta_{1}$, which ranges from -3 to 3 in Fig. 1 . Whether this bias has a large or negligible effect on $\hat{\beta}_{1}$ will depend on the magnitude of $\beta_{1}$ (e.g., a bias of 3 is very small if $\beta_{1}=100$ ). Because bias grows linearly with $\beta_{3}$, estimating whether omitted variable bias has any practical effect would require comparing $\beta_{1}$ and $\beta_{3}$. In PER, because we do not know the "true" model, we cannot compare these effect sizes, meaning we cannot evaluate the practical effects of omitted variables. We discuss practical strategies below, after considering a case of omitted variable bias with real data.

\section{ILLUSTRATIVE EXAMPLES OF OMITTED VARIABLE BIAS WITH REAL DATA}

In this section, we consider an intentionally simple scenario to demonstrate the effect of omitted variable bias using an authentic PER dataset. We use data collected with the Colorado Learning Attitudes about Science Survey for Experimental Physics (E-CLASS), which was designed to measure students' attitudes and beliefs about experimental physics [29], to investigate our hypothetical research question from Sec. I.

\section{A. Data sources}

The E-CLASS consists of 30 five-point Likert items where students indicate the extent of their agreement (from "strongly disagree" to "strongly agree") with statements such as, "When I am doing an experiment, I try to make predictions to see if my results are reasonable." Students are scored based on how well their responses align with responses from expert physicists: positive scores (maximum of 30) represent overall alignment with experts and negative scores (minimum of -30 ) represent misalignment with experts $[29,30]$.

To address our hypothetical research question, we collected pre- and post-test E-CLASS data from physics students at Cornell University and information about students' intended major. We collected data from classes that employed different types of lab pedagogies. Some labs were highly traditional, while other labs used nontraditional pedagogies (see Ref. [31]). In our illustrative examples, we also used information about students' underrepresented minority (URM) status. These variables were deliberately chosen to illustrate the effect of omitted variable bias (or lack thereof). We asked students to indicate their intended major at the end of the E-CLASS post-test, while students' URM status was provided by the registrar. The registrar classified students who identified as Black/African American, Hispanic, Native American, or Native Hawaiian as URM. As noted in Sec. I A, we only included 556 students who completed both the pretest and post-test in the analysis. We note, again, that we used complete case analysis in these examples. In more rigorous analyses, care should be taken to address the impacts of missing data on results through, for 
example, multiple imputation [5]. As stated in Sec. I A, effect sizes presented below are not meaningful and readers should instead focus on the methodological implications of our results.

\section{B. Methods}

Unlike in Sec. II, we do not know the "true" model when dealing with real data. We almost certainly violate our conditional ignorability assumption regardless of the model we use, but we cannot quantify the degree or even the direction of omitted variable bias. We instead examine the Pearson correlation coefficients between major, instruction, and URM status to identify the potential for omitted variable bias based on the cases examined in Sec. II.

We use three regression models to illustrate the effect of including or omitting different variables on our conclusions for our hypothetical research question. In all three models, we use students' E-CLASS post-test scores as the dependent variable, students' intended majors as an independent variable, and students' E-CLASS pretest scores as a covariate. In the first model, we use no additional control variables and simply investigate the effect of students' intended majors on E-CLASS post-test scores controlling for pretest scores. In the second model, we additionally control for the type of instruction students received. This model allows us to estimate to what extent the effect attributed to students' intended majors in model 1 could be attributed to the type of lab instruction students received. In the third model, we control for students' URM status instead of the type of lab instruction. This model allows us to estimate to what extent the effect attributed to students' intended majors in model 1 could be attributed to how society and our educational systems position people by race or ethnicity.

\section{Examples \\ 1. Model 1: Effect of major controlling for pretest scores}

For model 1, we fit a multiple linear regression model that controlled for students' pretest scores to determine the effect of students' intended major on their E-CLASS post-test scores, independent of pretest scores (Model 1 in Table I).

Our results indicate that, controlling for pretest scores, students who intend to major in physics score approximately 3.4 points higher on the E-CLASS post-test, on average, than students who intend to major in engineering or another science. This difference corresponds to roughly 0.399 post-test score standard deviations. We can interpret this coefficient through the following:

Given two students, one who intended to major in physics and another who intended to major in engineering, with identical E-CLASS pretest scores, we would expect, on average, the student who intended to major in physics to score 3.4 points higher on the post-test than the student who intended to major in engineering.

\section{Model 2: Effect of major controlling for pretest scores and type of instruction}

We extend on the first model by examining the role of lab instruction in interpreting the effect of students' intended majors on E-CLASS post-test scores. The Pearson correlation coefficient between major and instruction is 0.574 in our sample, a strong correlation that can lead to omitted variable bias in estimating the effect of major if instruction is omitted from our model (see line 4 in Fig. 1). We fit Model 2 in Table I to determine the independent effects of students' pretest scores, intended majors, and the type of lab instruction students received.

TABLE I. Results of fitted linear regressions for three illustrative examples using the E-CLASS. Models were built to explicitly illustrate omitted variable bias and are not based on any causal theory. Standardized coefficients are reported with pre- and post-test scores grand mean centered.

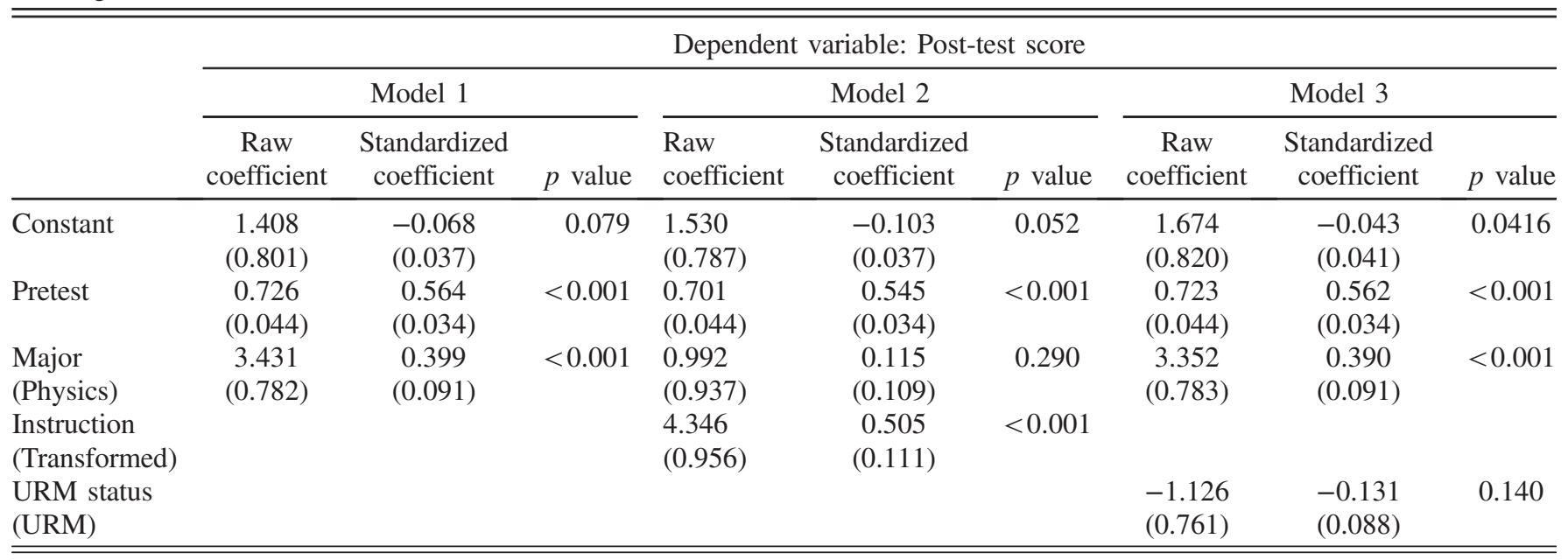


Our results indicate that, after controlling for pretest scores and the type of lab instruction that students received, the effect of intended major on post-test score is about 1.0 points (or 0.115 standard deviations). We can interpret this coefficient through the following:

Given two students, one who intended to major in physics and another who intended to major in engineering, with identical E-CLASS pretest scores and provided the same type of lab instruction, we would expect, on average, the student who intended to major in physics to score 1.0 points higher on the post-test than the student who intended to major in engineering.

Students' intended majors still have an effect on E-CLASS post-test scores in this scenario, but the size of this effect is $71 \%$ smaller than the estimated effect in model 1 . Much of the effect previously attributed to students' intended majors can be attributed to the type of lab instruction students received.

\section{Model 3: Effect of major controlling for pretest scores and URM status}

In our final model, we examine how accounting for students' URM status affects our interpretation of the effect of students' intended major on E-CLASS post-test scores. The Pearson correlation coefficient between major and URM status is -0.067 in our sample, a very weak correlation. Omitting URM status from our models will lead to omitted variable bias, but the magnitude of this bias is likely small (see line 1 in Fig. 1). We fit Model 3 in Table I to determine the independent effects of students' pretest scores, intended majors, and URM status.

Our results indicate that, after controlling for students' pretest scores and URM status, the effect of intended major on post-test score is about 3.4 points ( 0.390 standard deviations). We can interpret this coefficient through the following:

Given two students, one who intended to major in physics and another who intended to major in engineering, with identical E-CLASS pretest scores and the same race or ethnicity, we would expect, on average, the student who intended to major in physics to score 3.4 points higher on the post-test than the student who intended to major in engineering.

The estimated effect of students' intended major is only $2.3 \%$ smaller than the estimated effect in model 1 . The coefficient for URM status is nonzero, but the omission of URM status from model 1 does not noticeably affect our estimate of the effect of students' intended major on E-CLASS post-test scores.

\section{Summary}

Here we have demonstrated scenarios where omitted variables do (omitting lab instruction) and do not (omitting
URM status) affect conclusions. Using real data, we showed that omitting key variables from analyses can have large effects on estimated coefficients, while other variables can be safely ignored without affecting results. Whether omitting a particular variable will lead to bias depends on the strength of the effect of that variable on the outcome variable and on the correlations between the included and omitted variables. Because lab instruction was strongly correlated with students' major and affected their beliefs about experimental physics, omitting that variable led to a large bias. On the other hand, URM status was weakly correlated with students' major and had a small effect on students' post-test scores, so omitting URM status did not result in a bias.

A common concern during statistical modeling is that adding variables may sacrifice measurement precision in exchange for accuracy, particularly if the effect of the added variable is small. This is not necessarily the case: for example, the standard errors on the estimated effect of the major variable in models 1 and 3 are unchanged $(0.78$ in each case). The standard error on major in model 2 is different, but the estimated effect size also changed substantially.

\section{DISCUSSION}

In this study we explored how omitting variables in explanatory modeling can affect how we interpret the effects of other variables on student outcomes. We demonstrated this effect using analytical solutions and real data. In Sec. II, we showed how the magnitude of omitted variable bias depends on the "true" effects of omitted variables and the correlations between all variables. When the "true" effects of omitted variables are large and those omitted variables are strongly correlated with included variables, the size of effects for included variables are likely substantially biased.

The results of Sec. II allowed us to interpret our findings using real data in Sec. III through a lens of omitted variable bias. We found that lab instruction was strongly correlated with students' intended major and omitting lab instruction from our statistical model noticeably changed our estimate of the effect of major on students' E-CLASS post-test scores. We also showed that adding (or omitting) a variable only weakly correlated with students' intended major (students' URM status in this case) had no practical effect on either the size or the precision of the estimated effect of major. We demonstrated that including or excluding different variables in models can lead to different conclusions for the same research question.

We introduce omitted variable bias into our models whenever we violate the conditional ignorability assumption. Though we cannot check this assumption, we can safely assume that we violate it in any nonexperimental study (like the one we used here). The degree to which omitted variable bias will affect conclusions depends on the 
conditions mentioned above, but this bias cannot be measured because we would need to know the "true" model and measure unobservable variables. Nissen et al. made this point when examining the effects of missing data in PER [5], stating that "the boundary between [data] missing at random and missing not at random cannot be firmly established because doing so requires observing the unobserved data." Missing data are a specific form of violation of ignorability that affects conclusions.

Shmueli offer recommendations for model building and variable selection in explanatory modeling [2] that we reiterate here. Researchers should decide on the structure of the statistical model and whether to include variables in the model based on the theoretical causal model [2]. Researchers should also avoid "trimming potentially theoretically meaningful variables $[\ldots]$ unless one is quite certain that the coefficient for the variable is near zero, that the variable is inconsequential, and that trimming will not introduce misspecification error" $[2,32]$. As part of this recommendation, a researcher might choose to retain a variable in a model even if it is not statistically significant so long as there is a strong theoretical justification for including that variable in the model. In our illustrative example using real data, for example, we might choose to retain URM status in our model if we deemed it to be theoretically meaningful despite this variable not being statistically significant.

\section{CONCLUSIONS}

This study demonstrated why omitted variable bias is a problem that PER should be aware of and how this bias arises in statistical models. Because in PER we never know the "true" model and can never account for all possible variables, we are always prone to omitted variable bias. Whenever possible we should control for potential confounding variables that can have a large effect on the results of our studies. As argued elsewhere (see, e.g., Ref. [33]), as readers and authors, we must also entertain plausible alternative explanations when evaluating quantitative results.

\section{ACKNOWLEDGMENTS}

We wish to thank Shima Salehi, Eric Burkholder, and Danny Caballero for helpful feedback on this manuscript. This material is based on work supported by the National Science Foundation under Grant No. 1611482 and by Cornell University's College of Arts and Sciences Active Learning Initiative.
[1] A. Gelman and J. Hill, Data Analysis Using Regression and Multilevel/Hierarchical Models (Cambridge University Press, Cambridge, England, 2006).

[2] G. Shmueli, To explain or to predict? Stat. Sci. 25, 289 (2010).

[3] P. R. Rosenbaum and D. B. Rubin, The central role of the propensity score in observational studies for causal effects, Biometrika 70, 41 (1983).

[4] D. B. Rubin, Bayesian inference for causal effects: The role of randomization, Ann. Stat. 6, 34 (1978).

[5] J. Nissen, R. Donatello, and B. Van Dusen, Missing data and bias in physics education research: A case for using multiple imputation, Phys. Rev. Phys. Educ. Res. 15, 020106 (2019).

[6] D. B. Rubin, Inference and missing data, Biometrika 63, 581 (1976).

[7] M. Jaeger, Ignorability in statistical and probabilistic inference, J. Artif. Intell. Res. 24, 889 (2005).

[8] V. P. Coletta and J. A. Phillips, Interpreting FCI scores: Normalized gain, preinstruction scores, and scientific reasoning ability, Am. J. Phys. 73, 1172 (2005).

[9] K. Diff and N. Tache, From FCI To CSEM to lawson test: A report on data collected at a community college, in AIP Conference Proceedings (American Institute of Physics, New York, 2007), Vol. 951, pp. 85-87.

[10] P. Nieminen, A. Savinainen, and J. Viiri, Relations between representational consistency, conceptual understanding of the force concept, and scientific reasoning, Phys. Rev. ST Phys. Educ. Res. 8, 010123 (2012).

[11] B. A. Pyper, Changing scientific reasoning and conceptual understanding in college students, in AIP Conference Proceedings (American Institute of Physics, New York, 2012), Vol. 1413, pp. 63-65.

[12] V. P. Coletta and J. J. Steinert, Why normalized gain should continue to be used in analyzing preinstruction and postinstruction scores on concept inventories, Phys. Rev. Phys. Educ. Res. 16, 010108 (2020).

[13] R. Henderson, J. Stewart, and A. Traxler, Partitioning the gender gap in physics conceptual inventories: Force Concept Inventory, Force and Motion Conceptual Evaluation, and Conceptual Survey of Electricity and Magnetism, Phys. Rev. Phys. Educ. Res. 15, 010131 (2019).

[14] T. J. Nokes-Malach, Z. Y. Kalender, E. Marshman, C. Schunn, and C. Singh, Prior preparation and motivational characteristics mediate relations between gender and learning outcomes in introductory physics, in Proceedings of the Physics Education Research Conference 2018, Washington, DC (American Association of Physics Teachers, Washington, DC, 2018).

[15] S. Salehi, E. Burkholder, G. P. Lepage, S. Pollock, and C. Wieman, Demographic gaps or preparation gaps?: The large impact of incoming preparation on performance of 
students in introductory physics, Phys. Rev. Phys. Educ. Res. 15, 020114 (2019).

[16] B. R. Wilcox and H. Lewandowski, Research-based assessment of students' beliefs about experimental physics: When is gender a factor? Phys. Rev. Phys. Educ. Res. 12, 020130 (2016).

[17] L. McCullough, An overview on research on gender and under-represented ethnicities in physics education, in Getting Started in PER, edited by C. Henderson and K. A. Harper (American Association of Physics Teachers, College Park, MD, 2018).

[18] A. Madsen, S. B. McKagan, and E. C. Sayre, Gender gap on concept inventories in physics: What is consistent, what is inconsistent, and what factors influence the gap? Phys. Rev. ST Phys. Educ. Res. 9, 020121 (2013).

[19] R. Henderson, C. Zabriskie, and J. Stewart, Rural and first generation performance differences on the force and motion conceptual evaluation, in Proceedings of the Physics Education Research Conference 2018, Washington, DC (Ref. [14]).

[20] E. Brewe, V. Sawtelle, L. H. Kramer, G. E. OBrien, I. Rodriguez, and P. Pamelá, Toward equity through participation in Modeling Instruction in introductory university physics, Phys. Rev. ST Phys. Educ. Res. 6, 010106 (2010).

[21] J.E. Watkins, Examining issues of underrepresented minority students in introductory physics, Ph.D. thesis, Harvard University, 2010.

[22] B. V. Dusen, J.-S.S. White, and E. A. Roualdes, The impact of learning assistants on inequities in physics student outcomes, in Proceedings of the Physics Education Research Conference 2016, Sacramento, CA (American Association of Physics Teachers, Sacramento, CA, 2016), pp. 360-363.

[23] B. V. Dusen and J. Nissen, Systemic inequities in introductory physics courses: the impacts of learning assistants, in Proceedings of the Physics Education Research Conference 2017, Cincinnati, OH (American Association of Physics Teachers, Cincinnati, OH, 2017), pp. 400-403.
[24] B. Van Dusen and J. Nissen, Equity in college physics student learning: A critical quantitative intersectionality investigation, J. Res. Sci. Teach. 57, 33 (2020).

[25] J. Madden, S. Pandita, J. Schuldt, B. Kim, A. S. Won, and N. Holmes, Ready student one: Exploring the predictors of student learning in virtual reality, PLoS One 15, e0229788 (2020).

[26] K. A. Clarke, The Phantom Menace: Omitted variable bias in econometric research, J. Conflict Resolut. 22, 341 (2005).

[27] E. Burkholder, C. Walsh, and N. Holmes, Examination of quantitative methods for analyzing data from concept inventories, Phys. Rev. Phys. Educ. Res. 16, 010141 (2020).

[28] B. Van Dusen and J. Nissen, Modernizing use of regression models in physics education research: A review of hierarchical linear modeling, Phys. Rev. Phys. Educ. Res. 15, 020108 (2019).

[29] B. R. Wilcox and H. J. Lewandowski, Students' epistemologies about experimental physics: Validating the Colorado Learning Attitudes about Science Survey for experimental physics, Phys. Rev. Phys. Educ. Res. 12, 010123 (2016).

[30] B. M. Zwickl, T. Hirokawa, N. Finkelstein, and H. Lewandowski, Epistemology and expectations survey about experimental physics: Development and initial results, Phys. Rev. ST Phys. Educ. Res. 10, 010120 (2014).

[31] E. M. Smith, M. M. Stein, C. Walsh, and N. Holmes, Direct Measurement of the Impact of Teaching Experimentation in Physics Labs, Phys. Rev. X 10, 011029 (2020).

[32] J. Jaccard, Interaction Effects in Logistic Regression (SAGE publications, Thousand Oaks, CA, 2001).

[33] A. V. Knaub, J. M. Aiken, and L. Ding, Two-phase study examining perspectives and use of quantitative methods in physics education research, Phys. Rev. Phys. Educ. Res. 15, 020102 (2019). 